Accelerator Development Department

Accelerator Physics Division

BROOKHAVEN NATIONAL LABORATORY

Associated Universities, Inc.

Upton, NY 11973

Accelerator Physics Technical Note No. 30

Beam Abort in RHICAGR

A.G. Ruggiero

October 1991 


\title{
BEAM ABORT IN RHICAGR
}

\author{
A.G. Ruggiero \\ Accelerator Development Department \\ Brookhaven National Laboratory \\ October 1991
}




\section{Contents}

The Need for Long Contiguous Warm Spaces . . . . . . . . . . . . 1 What are we Aborting? .................. . . . . .

Internal Abort for RHICAGR . . . . . . . . . . . . . 4

External Abort from RHICAGR . . . . . . . . . . . . . 9

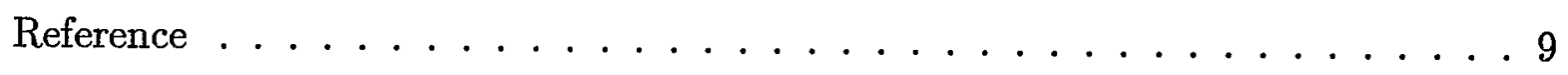




\title{
BEAM ABORT IN RHICAGR
}

\author{
A.G. Ruggiero \\ Accelerator Development Department \\ Brookhaven National Laboratory \\ October 1991
}

\section{The Need for Long Contiguous Warm Spaces}

The RHICAGR lattice described in the internal note AD/AP-26 (August 1991) does not provide for a long contiguous warm space. The design approach used optimizes the ease of tunability of the insertion telescope by increasing the distance between Q4 and Q5 and by placing these quadrupoles relatively closer to BC3 and Q3. The actual distance between Q4 and Q5 is $14.7 \mathrm{~m}$, between $\mathrm{Q} 4$ and $\mathrm{BC} 3$ is $14.8 \mathrm{~m}$ and between $\mathrm{BC} 3$ and Q3 is $5.3 \mathrm{~m}$. Thus there is an overall drift space for a total of $34.8 \mathrm{~m}$ between Q3 and Q5 but broken by the presence of $\mathrm{BC} 3$ which is $3.4 \mathrm{~m}$ long and $\mathrm{Q} 4$ which is $2.25 \mathrm{~m}$ long. All this is shown again in some details in Fig. 1.

Recently a committee ${ }^{1}$, called to express judgment on the fitness and performance of the RHIC lattices, has highly recommended the need for long contiguous warm spaces in RHIC. I would like to question here the motivations for this need and the length of the warm spaces required. In my opinion, the committee was not very specific about these issues. There is a very vague statement like "... In general, long contiguous free spaces are always valuable. Below we address specifically the injection/ejection considerations...". The committee report then goes on describing and judging the merits and defaults of the injection and ejection schemes proposed. In particular, the point which is of my concern here relative to RHICAGR is “... only very preliminary design approaches were presented, and the suggested solutions could be readily improved upon conceptually. Nevertheless, taking these potential improvements into account, the possibility of acceptable technical execution of the designs presented must be regarded as problematic ..." I would like to 
$25-$

$m^{1 / 2} \sqrt{\beta}$

$20-$

$15-$

$10-$

Figure 1: RHICAGR Insertion.

N

$$
\leftarrow 630 \mathrm{~m}
$$

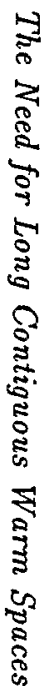


capitalize on the first part of this statement and challenge the conclusion implied in the second part. I will show in this report that actually a closer re-examination of the beam abort from RHICAGR suggests a solution which requires a shorter abort kicker with an extraction angle of less than $1 \mathrm{mrad}$. Moreover, the scheme can be fit very well in the RHICAGR proposed quadrupole configuration and takes full advantage of the presence of the Q4 quadrupole.

The only other reason suggested in the committee report for the requirement of "...> $30 m$ of magnet-free space in the insertion is to provide for the possible option to operate RHIC with polarized protons ...". Possible option?... polarized protons?. . I could argue with this argument with a long list of: why? when? how? who?... In my opinion it is a too vague motivation for the RHIC project. Yet, the report concludes: "... with spaces between magnets, necessary so that the snake produces zero net deflection, each snake requires altogether about 20-25m of free space if the magnets are warm at 1.5 T. It is conceivable that a quadrupole within the snake may be allowable, but this is an extra complication, preferably to be avoided..." It appears to me, at a closer look, that actually the complication is minute. ${ }^{*}$ The quadrupole arrangement in the insertion must be determined from other important considerations as explained in AD/AP-26.

\section{What are we Aborting?}

Now I would like to address some sources of confusion. First of all there are two choices internal abort and external abort; I am afraid that the committee has made some not relevant comparisons between the several methods proposed. In AD/AP-26 I have described a complete one-turn external extraction system with a $10 \mathrm{~m}$ long $4 \mathrm{kG}$ kicker magnet for a total bending angle of $4.7 \mathrm{mrad}$ at maximum energy. I am aware of the difficulty and cost of this device. Nevertheless all the other proposals deal with an internal abort approach to start, which later can be expanded to a fully external abort. It is very easy to accommodate an internal abort system in RHICAGR, and I would like to challenge the statement advanced by somebody that one needs at least $40 \mathrm{~m}$ space free of magnets to accommodate it. Actually this drift is too long and later can actually be an impediment to upgrade to a fully external abort system.

* A report on this subject will follow soon. 
Another source of confusion is the distinction between betatron emittance and betatron acceptance. The former describes the beam dimensions and depends on the beam energy and on other beam properties; the latter is an amount of space available which has nothing to do with what the beam does or even if the beam exists. The betatron acceptance for RHIC is $6 \pi \mathrm{mm} \cdot \mathrm{mrad}$ at all energies and in all conditions; it is the result of a combination of dynamical and physical aperture, mostly set by BC2, Q2 and Q3. There is no such thing as a normalized acceptance! The beam actual beam emittance is to be smaller than the acceptance at all energies; if it is larger, part of it is lost. Actually in RHIC the full beam emittance is always considerably smaller than the acceptance at any energy from injection to top energy.

The requirement on the abort system, to play safe, is to be capable to abort the RHIC betatron acceptance which we have seen to be $6 \pi \mathrm{mm} \cdot \mathrm{mrad}$ at any energy. This will allow to disregard the actual beam position within the allowable aperture and to be on the bullseye at any time.

\section{Internal Abort for RHICAGR}

The internal abort system which I propose for RHICAGR is shown in Fig. 2. A vertical kicker is located between Q3 and BC3, with an effective length of $3 \mathrm{~m}$ and a field of $2.2 \mathrm{kG}$ at top energy. The bending angle is then $\theta_{k}=0.8 \mathrm{mrad}$. The deflected beam goes through BC3 with less than $5 \mathrm{~mm}$ vertical displacement (downward) at the exit of the magnet. The distance between the center of the kicker and the center of the Q4 quadrupole is $d_{1}=21.9 \mathrm{~m}$. A collimator/absorber is located between Q4 and Q5; it is $4 \mathrm{~m}$ long; there is a drift of $3 \mathrm{~m}$ between the end of the collimator and Q5; and the distance between the center of Q4 and the front of the collimator is $d_{2}=8.8 \mathrm{~m}$.

Due to kicker bending the beam arrives at Q4 displaced by $s_{1}=d_{1} \theta_{k}=17.5 \mathrm{~mm}$. The Q4 quadrupole is defocusing vertically and provides an extra kick which deflects the beam more downward

$$
\Delta \theta_{Q 4}=\left(\frac{B^{\prime} \ell}{B \rho}\right) s_{1}
$$

For the $\beta^{*}=2 \mathrm{~m}$ operation $B^{\prime} \ell / B \rho=0.125 \mathrm{~m}^{-1}$ and $\Delta \theta_{Q 4}=2.4 \mathrm{mrad}$. Thus the presence of $\mathrm{Q} 4$ at that location I consider beneficial since it actually provides most of the 


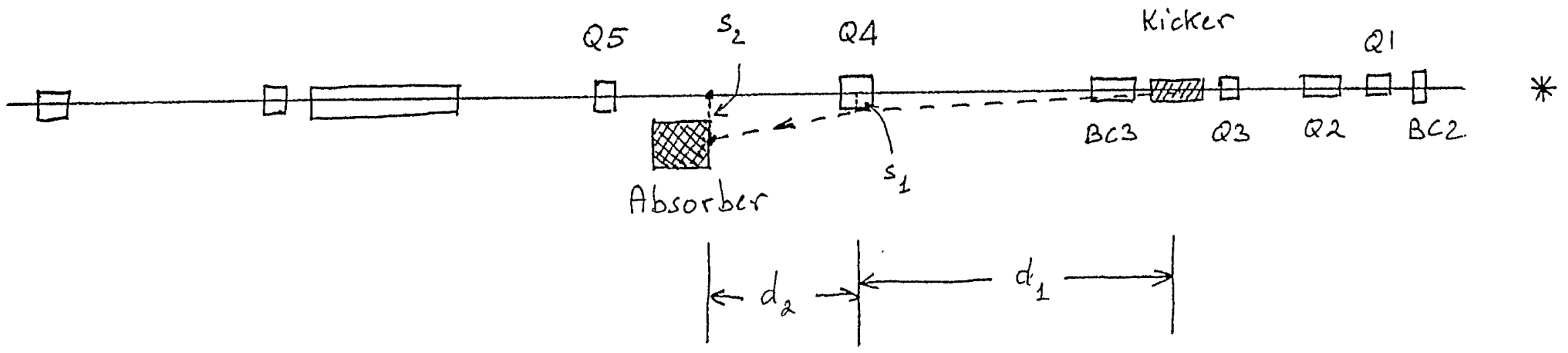


beam deflection. The quadrupole has a large aperture with a coil bore of $130 \mathrm{~mm}$ and thus the displacement of $17.5 \mathrm{~mm}$ is of no consequence. A complication may arise from an accidental misfiring of the kicker during injection where the beam energy is lower; if the accidental misfire generates the maximum kicker field, the kick angle can be as large as $6.5 \mathrm{mrad}$ and the displacement at Q4 at large as $140 \mathrm{~mm}$. In this case clearly the beam may hit the walls of Q4 and cause a quench. There are ways to control these consequences by either putting a cap on the kicker electric power supply or by preceding Q4 with a collimator/absorber to intercept the beam at low energy. Beyond this I do not foresee other problems.

The beam displacement at the front of the collimator is then

$$
\begin{aligned}
s_{2} & =\left(d_{1}+d_{2}+0.135 d_{1} d_{2}\right) \theta_{k} \\
& =(56.7 \mathrm{~m}) \theta_{k}
\end{aligned}
$$

For $\theta_{k}=0.8 \mathrm{mrad} \mathrm{I}$ derive $s_{2}=45 \mathrm{~mm}$.

There is a slow continuous reduction of the gradient of Q4 when the insertion is tuned from $\beta^{*}=2 \mathrm{~m}$ up to $\beta^{*}=16 \mathrm{~m}$. At the upper end $\left(B^{\prime} \ell / B \rho\right)=0.10 \mathrm{~m}^{-1}$. To compensate for this reduction, the kicker field is to be raised by about $10 \%$ to provide $\theta_{k}=0.9 \mathrm{mrad}$. In reality large $\beta^{*}$ operation may be required only during injection when the strength of the kicker is not a concern.

Figure 3 shows the location of the circulating and aborted acceptance $(6 \pi \mathrm{mm} \cdot \mathrm{mrad})$ at Q4 for $\beta^{*}=2 \mathrm{~m}$. There is no significant change for larger values of $\beta^{*}$.

Figure 4 demonstrates the location of the same circulating and aborted betatron acceptance at the front and back of the absorber block for $\beta^{*}=2 \mathrm{~m}$. Again, (a nice property of the RHICAGR lattice), the geometry has only a weak dependence with the value of $\beta^{*}$. The separation of the block inner edge from the collider mid-plane is taken to be $20 \mathrm{~mm}$, which matches the collider betatron acceptance.

Finally the region between the kicker and the absorber is dispersion free which can also be regarded a nicety. 
8

Internal Abort for RHICAGR

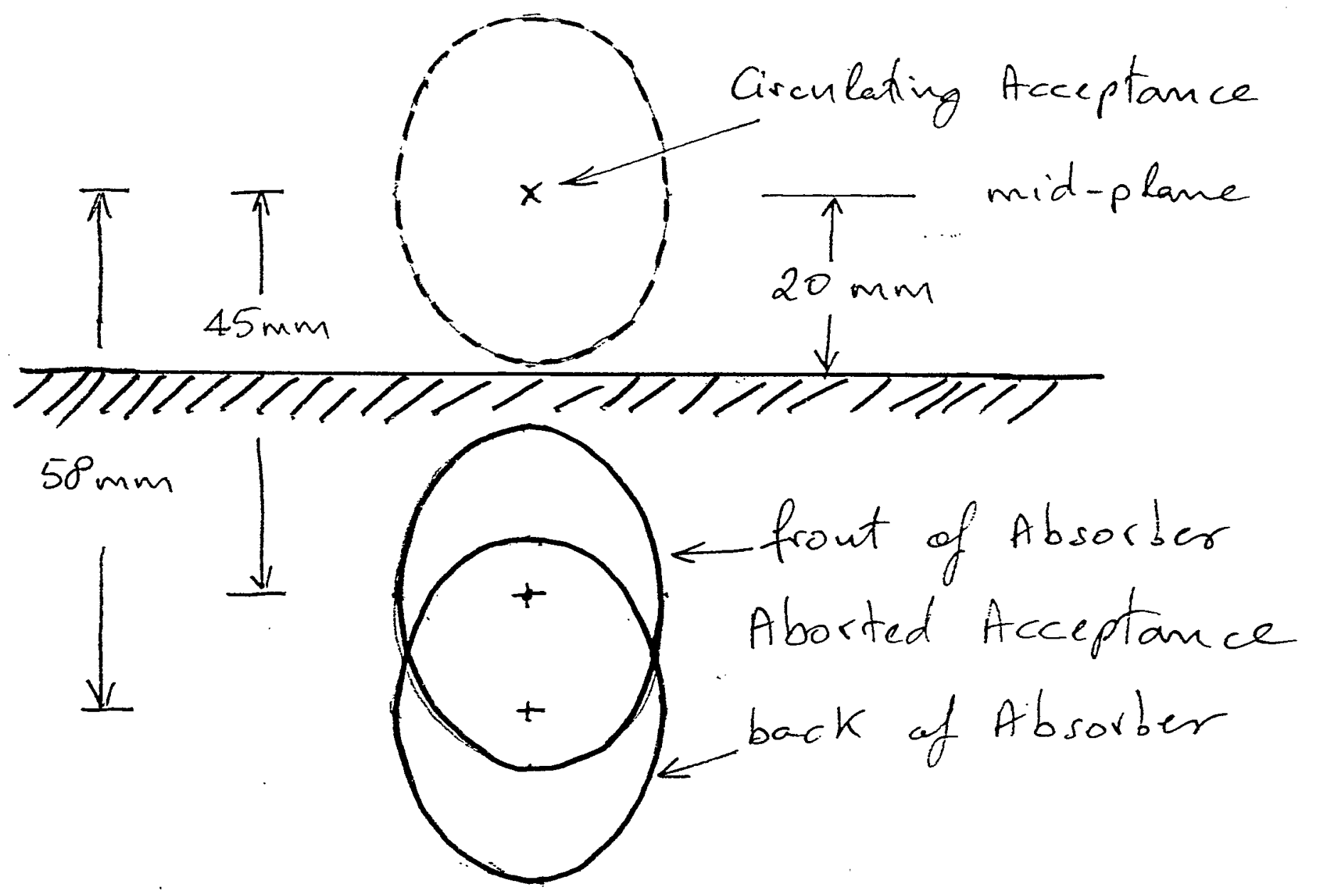

Pic Betatron Acceptance $=6 \pi$ mm in rad

Figure 4: Circulating and Aborted Acceptance at the Absorber $\left(\beta_{H}=20\right.$ $35 \mathrm{~m}, \beta_{V}=60-70 \mathrm{~m}$ for $\beta^{*}=2 \mathrm{~m}$ ). 


\section{External Abort from RHICAGR}

It is relatively easy to upgrade the internal abort system described above to an external abort. This can be accomplished with the addition of a $10 \mathrm{~m}$ longer kicker to the other side of $\mathrm{BC} 3$ with the same field of $2.2 \mathrm{kG}$ and of a septum extraction magnet between Q4 and Q5. The central point of the kicker can be taken now at a distance $d_{1} \sim 10 \mathrm{~m}$ from the middle of $\mathrm{Q} 4$, and the total kick angle is now increased to $4 \mathrm{mrad}$. The beam displacement at $\mathrm{Q} 4$ becomes $s_{1}=40 \mathrm{~mm}$ with the actual location of the betatron acceptance shown again in Fig. 3 by the dotted ellipse. The extra deflecting kick angle due to Q4 is now even larger, $5 \mathrm{mrad}$ for a total of $9 \mathrm{mrad}$ at the exit of $\mathrm{Q} 4$. About one meter after the end of Q4, that is at the entrance of the septum magnet, the total beam displacement is now 59 $\mathrm{mm}$. The septum magnet which follows is $12 \mathrm{~m}$ long with a field of 1 Tesla. Basically the external abort here described follows very closely the one proposed in AD/AP-26 except for the addition of the $3 \mathrm{~m}$ longer kicker between Q3 and BC3 which lowers the field to a more manageable level.

\section{Reference}

1. A. Chao, Report of the RHIC Lattice Review Committee, Sept. 1991. 